\section{LA SOCIEDAD CIVIL Y LAS ENFERMEDADES RARAS}

\author{
Mercedes Serrano \\ Hospital Sant Joan de Déu \\ ORCID iD: https://orcid.org/0000-0002-2056-2428
}

mserrano@hsjdbcn.org

\section{CIVIL SOCIETY AND RARE DISEASES}

Cómo citar este artículo/Citation: Serrano, M. (2018). La sociedad civil y las enfermedades raras. Arbor, 194 (789): a459. https://doi.org/10.3989/arbor.2018.789n3001

Recibido: 30 abril 2015. Aceptado: 13 mayo 2016.
Copyright: (C) 2018 CSIC. Este es un artículo de acceso abierto distribuido bajo los términos de la licencia de uso y distribución Creative Commons Reconocimiento 4.0 Internacional (CC BY 4.0).
RESUMEN: Las enfermedades poco frecuentes suelen ser las grandes olvidadas tanto de los sistemas sanitarios como de la investigación. Esto da lugar a que los pacientes y los familiares encuentren dificultades no solo para recibir una asistencia médica adecuada, sino también para conseguir un verdadero apoyo psicosocial. No obstante, se están produciendo cambios muy positivos, como son la creciente presencia del conjunto de las enfermedades raras en las políticas nacionales e internacionales, la evolución en el papel que adoptan los pacientes, los médicos y los investigadores y en sus relaciones mutuas, la creciente sensibilización e implicación social, las oportunidades crecientes de difusión y de sensibilización y la circunstancia favorable desde el punto de vista tecnológico y de comunicación. La complejidad de estos cambios y la responsabilidad que cada grupo de agentes implicados está asumiendo en este nuevo marco son asuntos controvertidos y están surgiendo cuestiones de índole ética. A veces no es fácil responder a ellas y solo se puede explicar el papel que adoptan los distintos agentes por imperiosa necesidad.

PALABRAS CLAVE: Enfermedades raras; empowerment; medicamentos huérfanos; asociaciones de pacientes.
ABSTRACT: Rare diseases are often neglected from both health systems and research. The situation for patients and families is characterized by difficulties not only to receive adequate medical care, but also to get a necessary psychosocial support. However, remarkable positive changes are taking place, such as the growing presence of rare diseases as a group in national and international policies, the changing roles of patients, physicians and researchers and their mutual relations, increasing awareness and social involvement, greater opportunities for dissemination and awareness and the current favourable communications and technological environment. The complexity of these changes and the responsibilities that each group of stakeholders should take in this new framework is controversial and questions of an ethical nature are arising. These are often difficult to answer and the role adopted by the different agents can be explained by their pressing needs.

KEYWORDS: Rare diseases; empowerment; orphan drugs; patient societies. 
Las enfermedades raras incluyen un grupo muy diverso de enfermedades, el número de las cuales se estima que podría oscilar entre las 5.000 y las 7.000 . Resulta difícil de concretar este dato, dado que cada mes aparecen nuevas enfermedades vinculadas a nuevas mutaciones y nuevos genes, que hasta ahora no habían sido descritos. Sirva de ejemplo el siguiente dato, relacionado con el mes de septiembre de 2014, en el que según el boletín de Orphanet, se habían descrito trece nuevas mutaciones o nuevos genes $y$ tres nuevos síndromes: http://www.orpha.net/actor/ EuropaNews/2014/140930.html\#NewSynd.

Las enfermedades poco frecuentes suelen ser las "grandes olvidadas" tanto de los sistemas sanitarios como de la ciencia en general, por la poca frecuencia con la que se manifiestan entre la población. Este aspecto hace complejo su abordaje, tanto desde el ámbito médico como desde el social. Nos encontramos ante un círculo vicioso, ante el denominado $p a-$ radigma de la rareza: al existir pocos pacientes de una misma enfermedad, no se fomenta la investigación y, por tanto, como no se estudia dicha enfermedad, es difícil el correcto abordaje médico ante la carencia de información tan básica como pueden ser los síntomas para su diagnóstico o la información relacionada con su evolución.

En el $80 \%$ de los casos estas enfermedades están vinculadas con alguna alteración genética, que se suele manifestar en el mismo momento del nacimiento o en los primeros años de la infancia. Este aspecto hace que mayoritariamente afecten a niños, implicando una cronicidad de las mismas, ya que muchas de ellas no tienen tratamiento curativo.

El principal criterio para considerar una enfermedad rara o huérfana es la prevalencia de la misma, es decir, el número de afectados respecto a la población en general. En la Unión Europea además también se consideran como factores asociados y necesarios la inexistencia de tratamientos adecuados o la severidad de los mismos. La Comisión Europea de Salud Pública califica como raras o poco frecuentes a aquellas "enfermedades, incluidas las de origen genético, que son crónicamente debilitantes o potencialmente mortales y que tienen tan poca prevalencia que se necesitan esfuerzos especiales combinados para combatirlas" (http://ec.europa.eu/health/rare_diseases/ policy/index_es.htm).

En Europa para que una enfermedad sea categorizada como rara debe afectar a menos de cinco personas por cada 10.000. En Estados Unidos la Ley de las enfermedades raras del 2002 define una enfermedad rara exclusivamente con relación a su prevalencia, señalando que es "cualquier enfermedad o condición que afecte a menos de 200.000 personas en los Estados Unidos" (http://history.nih.gov/research/downloads/PL107-280.pdf). Otros países utilizan otros parámetros para identificar la prevalencia máxima para que una patología pueda ser considerada como poco frecuente (Japón o Italia). Considerando el número de afectados, se estima que aproximadamente unos 30 millones de europeos y unos 25 millones de norteamericanos padecen una enfermedad rara.

La principal problemática asociada a las enfermedades poco frecuentes es la falta de conocimiento biomédico de las mismas, aspecto que retrasa su diagnóstico y que influye de forma directa en la calidad de vida de los afectados, sometiéndolos a un sufrimiento por la incertidumbre, que de otro modo podría ser, al menos en parte, evitado. La Federación Española de Enfermedades Raras, en el Estudio EnSerio, (Huete García et al., 2009) recoge un dato significativo en relación con el tiempo que transcurre entre la aparición de los síntomas y la obtención del diagnóstico confirmado de la enfermedad. En el caso de España, el promedio de tiempo que trascurre hasta la obtención del diagnóstico se sitúa entre cinco y siete años. En el $21,01 \%$ de los pacientes incluso llega a ser de diez o más años.

Unido a la carencia de conocimiento científico, hay que destacar también un importante desconocimiento del impacto y de la carga (emocional, familiar, social ) que supone una enfermedad poco frecuente, así como del tipo de discapacidades que conllevan con el tiempo. Este componente tan característico de las enfermedades poco frecuentes influye en el paciente y en su familia, haciendo que la afectación psicosocial de las mismas deba formar parte del enfoque integral del propio tratamiento (Kaplan et al., 2004).

En los últimos años, y sobre todo a raíz de la celebración en nuestro país durante 2013 del Año Nacional de las Enfermedades Raras, se están impulsando políticas específicas para un tratamiento diferenciado de estas patologías, respecto a las que, desde el punto de vista epidemiológico, permiten el estudio e intervención con un mayor número de pacientes. Diversos países de la Unión Europea, entre ellos España, se han adherido al Europlan, un proyecto liderado desde la Comisión Europea para la promoción de planes nacionales y estrategias especializadas en el ámbito de las enfermedades poco frecuentes (http://www.europlanproject.eu/Default). Este proyecto comporta el 
establecimiento de mesas de trabajo, integradas por diferentes perfiles de expertos, entre los que se encuentran los propios pacientes que son quienes mejor pueden defender sus derechos y necesidades.

Hay que destacar que las estrategias sanitarias y sociales pueden llegar a ser muy dispares entre países. Sirva de ejemplo la obligatoriedad de indexar cada uno de los pacientes afectados por una enfermedad poco frecuente en un registro nacional. Este instrumento es básico para poder conocer la prevalencia real de una enfermedad en un determinado país, así como los condicionantes propios del paciente (edad, herencia o mutación de novo, antecedentes familiares, etc.) y promover la investigación en relación con una determinada enfermedad e incluso organizar los recursos asistenciales. En nuestro país, a día de hoy, resulta imposible poder obtener datos fiables respecto al número de afectados de una enfermedad, pese a los esfuerzos que se están haciendo en las políticas públicas para promover el registro de los pacientes. Consideramos que la obligatoriedad, por el bien de paciente, de su familia y de la propia comunidad científica, debería ser el principal mecanismo para poder ir construyendo progresivamente un registro de pacientes que refleje del modo más fidedigno posible la realidad.

En relación con la comunidad médica, sigue habiendo un cierto olvido hacia estas enfermedades, que se ha correspondido, además, con un cierto olvido también por parte de la industria y de la investigación farmacéutica. Hablamos de enfermedades huérfanas con tratamientos huérfanos. Si defendemos una sanidad universal y solidaria, los pacientes con enfermedades raras tienen derecho a un conocimiento médico adecuado, a un diagnóstico rápido y a una investigación y tratamiento de primera línea (Editorial, 14 de junio de 2008). El ritmo de aprobación de fármacos de uso común es habitualmente más de diez veces mayor que el de fármacos huérfanos. Pero hay un intento de que esta situación cambie. En el año 2013 el International Rare Diseases Research Consortium (IRDiRD) se comprometió a aprobar al menos 200 fármacos huérfanos en el periodo 2013-2020, estableciendo este indicador como uno de sus objetivos generales de su plan de acción (http://www.irdirc.org). Y es que, por fortuna, a día de hoy, la investigación básica y clínica y la asistencia médica a este grupo de pacientes se ha convertido en una prioridad para los programas de financiación tanto en Europa como en Estados Unidos. Pero el camino es largo y tortuoso. La falta de información médica, la ausencia de terapias efectivas, la escasa investigación, las dificultades en la cobertura de la asistencia médica requerida como avanzábamos antes son algunos de los enemigos de este grupo de pacientes. Otra de las percepciones de los pacientes es una necesidad inminente de una mayor sinergia entre profesionales, lo que a veces topa de frente con la sensación de propiedad del paciente. Esta percepción de propiedad, en palabras de los propios pacientes, debe cambiar rotundamente para avanzar junto con el enfoque holístico e integral de cada caso.

Respecto a las percepciones de los pacientes y familiares es destacable el dato de que el $40 \%$ de las familias percibe que se podía haber diagnosticado de forma más precoz la enfermedad rara de su hijo y que las visitas a diferentes médicos han sido excesivas antes de llegar al diagnóstico definitivo (Anderson, Elliot y Zurynski, 2013). Esta demora puede estar vinculada a la falta de la descripción de la sintomatología de la enfermedad (muchas veces se trata de enfermedades con un amplio espectro de manifestaciones clínicas) y de la historia natural de la enfermedad, derivaciones a especialistas que no pueden aportar información etc., pero ello no debe ser óbice para generar interés médico en el conocimiento de todas aquellas enfermedades que aún siguen siendo grandes desconocidas para la comunidad científica.

Este retraso en el diagnóstico puede, en muchas ocasiones, condicionar un retraso en el inicio del tratamiento, la realización de pruebas complementarias molestas e innecesarias $y$, sin ninguna duda, un sufrimiento y tensión emocional evitables (tanto para el propio paciente como para su familia). Parece evidente que se requiere una mayor inversión en formación de los profesionales de la salud, un mayor estado de alerta frente a este grupo de enfermedades, y el desarrollo de nuevos recursos para su estudio clínico y de cuidados de forma integral al paciente y la familia.

El desarrollo de terapias y medicamentos huérfanos, como en otros casos, requiere unas fases de investigación clínica y básica previas al propio proceso de desarrollo y ensayo de los medicamentos, que de por sí tienen una duración media mínima en conjunto de unos diez años. El primer elemento para el mejor conocimiento de una enfermedad poco frecuente es lo que en medicina se denomina historia natural de la enfermedad. Se entiende por historia natural el preciso conocimiento de los síntomas para un correcto diagnóstico, así como su evolución en el tiempo para el correcto consejo e intervención médica, y además para posibilitar la valoración y evaluación del efecto de la intervención médica y para poder mesurarla. A par- 
tir de aquí la lucha debe basarse en el trabajo coordinado, tanto clínico como básico, para indagar posibles opciones terapéuticas que mejoren las condiciones de vida del paciente, el desarrollo de herramientas de medida y evaluación clínica para esas intervenciones $y$, en definitiva, para minimizar el desarrollo de la enfermedad o ralentizar sus efectos.

Como comentábamos con anterioridad, en la definición de enfermedad rara de la Unión Europea se contempla, además de la prevalencia de la enfermedad, la alteración que supone de la calidad y la esperanza de vida, así como el impacto familiar. Además, dado que se calcula que un $80 \%$ de las enfermedades raras tiene una causa genética, no podemos olvidar que en muchas familias las padece más de un paciente, con el subsiguiente incremento de las dificultades. A esto hay que añadir, desde el punto de vista no estrictamente médico, que los pacientes con enfermedades raras tienen la peor experiencia, entre el resto de los pacientes, sobre oportunidades sociales y económicas y, por supuesto, sobre cuidados médicos (Van Weely y Leufkens, 2004). En el caso de los pacientes pediátricos esto se manifiesta en forma de problemas en el rendimiento y en la adaptación escolar, en la autoestima y en la interacción social (Henderson, Packman y Packman, 2009). Refiriéndonos al impacto psicosocial de las enfermedades raras, en el Estudio socio-sanitario en Enfermedades Raras (ERES) realizado en 2007 en España se advierte que, entre los pacientes, hay una discapacidad percibida moderada o importante en el área emocional $(49,6 \%)$ así como en el área social $(48,3 \%)$ entre los encuestados (Avellaneda Fernández et al., 2007), independientemente del tipo de enfermedad. El 74\% de los encuestados tenían algún tipo de dependencia, denotando un gran problema de índole socio-sanitaria. Como consecuencia, una de las conclusiones del estudio es que la única forma de afrontar los complejos problemas de las personas afectas por enfermedades raras es un abordaje interdisciplinario e integral que incluya no solo los organismos políticos y administrativos sino también las asociaciones de pacientes, que son la principal fuente de apoyo e información, tal y como se muestra en este estudio, para la mayoría de los pacientes. Cada vez son más las instituciones sanitarias que, en muchos casos con la colaboración de las asociaciones de pacientes y sus comités científicos, contemplan el abordaje de las enfermedades poco frecuentes mediante unidades interdisciplinarias. Destacamos el impulso que se está dando en la Unión Europea al estudio y conocimiento del abordaje médico y científico de las enfermedades raras desde las instituciones sanitarias de los diferentes países miembros. Actualmente el Eu- ropean Union Committee of Experts on Rare Diseases (EUCERD) está llevando a cabo un estudio de campo cuantitativo y cualitativo para la definición de un mejor modelo asistencial que contemple una atención integral al paciente y a la familia afectada por una enfermedad poco frecuente.

Desde la época del Aceite de Lorenzo, uno de los más conocidos casos de familias que determinan un cambio en la investigación y terapias para la enfermedad de su hijo, han sido mucho los ejemplos de cómo las familias de los pacientes con enfermedades raras y los mismos pacientes son capaces de modificar el curso de la investigación y las posibilidades terapéuticas. No obstante, necesitamos reflexionar sobre si debe ser esa la verdadera función de las familias y asociaciones o si, por otra parte, se trata de una forma de suplir una necesidad no resuelta por los sistemas sanitarios actuales. ¿Es justo que las asociaciones financien la investigación? Estamos asistiendo a una creciente implicación de las asociaciones, muchas veces de forma independiente de su tamaño, que consiguen una gran movilización social para recaudar fondos y poder así financiar y fomentar una investigación que resulta insuficiente. Pero la pregunta de muchas de ellas es: ¿dónde está el límite?, ¿es esa su verdadera responsabilidad?

Lo que sí es indudable es su necesidad, porque la investigación en posibles terapias es la mayor fuente de esperanza para estas familias. Ahora bien, ¿están las asociaciones preparadas para seleccionar qué tipo de investigación y qué investigadores son la mejor opción? La realidad es que percibimos cada vez una mayor profesionalización de las asociaciones de pacientes, de forma que no depende tanto del número de familias afectadas por la enfermedad cuanto de las aptitudes y creencias personales de quienes las configuran y de sus habilidades profesionales utilizadas en pro de la asociación. Los comités científicos, que resultan el nexo de unión entre familias e investigación, parecen cada vez más necesarios si admitimos como adecuada esa función de sostén de la investigación de las asociaciones. El asesoramiento a las familias, la evaluación del correcto desarrollo de los proyectos, el acompañamiento en las decisiones... son algunas de las deseadas funciones de los comités científicos.

El poder y la voz de los pacientes es cada vez mayor y determinan políticas en apoyo de la investigación y de otros fines vinculados a las necesidades del colectivo (afectados y familias). Considerando el reducido número de pacientes por país de determinadas enfermedades poco frecuentes y el alto número de estas a nivel global, así como el tiempo necesario para el 
desarrollo de un proyecto de investigación parece más que necesaria la implicación de las familias en la promoción de la investigación. Está claro que la investigación es la esperanza para una mejor calidad de vida de los pacientes, y que en las enfermedades más discapacitantes el tiempo obra en contra del estudio y del desarrollo de agentes terapéuticos. Es por este motivo por lo que las asociaciones de pacientes en muchos casos son el primer foco para la promoción de la investigación en enfermedades raras. Conseguir la descripción de la historia natural de la patología, el fomento del estudio básico de la enfermedad y otras vías terapéuticas desde las asociaciones puede ser un gran paso para poder conseguir en el futuro financiación pública o incluso inversión de la industria farmacéutica ante la evidencia de los avances preliminares que se hayan podido conseguir.

Las asociaciones de pacientes se convierten además en el principal recurso de apoyo para sus propios miembros. El intercambio de experiencias entre pacientes/ familias recién diagnosticadas y aquellas que podríamos calificar de "veteranas" es de incalculable valor. El apoyo psicológico entre iguales facilita la mejor asimilación de las circunstancias vinculadas a la enfermedad, y es una vía imprescindible para unir fuerzas en pro de la mejor calidad de vida del paciente y de su familia. Dicho trabajo entre iguales permite a su vez contemplar los diferentes ángulos de visión para el tratamiento de la enfermedad (educación, recursos terapéuticos, investigación etc.). Las asociaciones de pacientes son un recurso asistencial que los médicos, y por extensión las instituciones hospitalarias, no deben olvidar y con las cuales es positivo un trabajo colaborativo. Podríamos considerar que las asociaciones de pacientes, deben ser el "primer medicamento" que debería "recetar" el especialista en el caso de aquellas enfermedades que no tienen tratamiento curativo. En muchos casos es la única vía terapéutica, con gran efecto positivo en el paciente y en la familia.

La situación económica actual de crisis ha despertado en muchos ciudadanos antes ajenos la solidaridad, y en esta coyuntura parece haber una mayor sensibilidad y compromiso social por las enfermedades raras. Las labores de sensibilización y difusión son cada vez más facilitadas por el avance de las tecnologías de la información y la comunicación (TIC). Las redes sociales, los medios de comunicación, que ya en el informe de Bañón Hernández, Fornieles Alcaraz, Solves Almela y Rius Sanchis (2011) resultaban más sensibles a las noticias sobre enfermedades raras, suponen un escaparate de excepción para llegar a más y más gente.
Pero una vez más, es difícil establecer un límite de la exposición de personas y familias que facilite la implicación social sin caer en el morbo. Por otro lado, la disponibilidad y la facilidad de acceso a información relevante para la familia a través de las TIC facilita una necesaria migración de la actividad médica a los propios domicilios de los pacientes para estar presentes en su día a día, como lo está la enfermedad y las dificultades. Un trabajo a veces invisible que modifica de forma significativa el empowerment de las familias y su bienestar en el más amplio sentido y que, en muchos casos, es más realizado por las asociaciones y poco asumido por las instituciones sanitarias.

Por suerte, existen cada vez más iniciativas sanitarias destinadas a formar y a asesorar al paciente, a la vez que a tratar cualquier duda que le pueda generar inseguridad a él o a su familia en el transcurso de la enfermedad. Los proyectos vinculados a lo que se viene denominando eHealth, se centran en el potencial de las nuevas tecnologías y en la recentralización de las prioridades de la investigación en las verdaderas necesidades de los pacientes. Conjugar estos dos aspectos está permitiendo el desarrollo de actuaciones con un alto impacto geográfico (las redes de comunicaciones no entienden de fronteras), que a su vez generan un efecto psicológico y social positivo en el paciente y en su familia. La confianza que inspiran las instituciones sanitarias que avalan muchas de estas iniciativas es el principal elemento que deben considerar los pacientes y sus familias a la hora de participar en las mismas. El consejo médico en el uso de las mismas debería hacerse y tener el mismo impacto que un fármaco en los hábitos de cuidado que debe incorporar y seguir el paciente.

Actualmente es más que evidente el cambio que se está dando en la relación médico-paciente. Podemos decir que estamos viviendo un cambio en la relación hacia una corresponsabilidad (responsabilidad compartida en la toma de decisiones), un acto médico donde tanto el paciente (o sus cuidadores en el caso de menores) como el médico deciden qué es lo mejor para el paciente en un proceso de negociación, de valoración de situaciones sociales, tiempos, costesbeneficios En este cambio de roles los pacientes con enfermedades raras han resultado pioneros para el cambio ya que, en ocasiones, la información con la que cuenta el médico, responsable de conocer las características de cientos de enfermedades, puede ser menor que la que llega a tener el paciente (o el cuidador) que se enfrenta a una sola enfermedad y con un estrecho vínculo emocional y personal. 
Pero el cambio en esta relación va también reflejándose en la relación del investigador y el paciente. Cada vez más las asociaciones de pacientes reclaman una cercanía de los investigadores, explicaciones comprensibles sobre en qué se basa su trabajo, y de forma recíproca una implicación formal en el reto de facilitar, en un momento de crisis económica como el que vivimos, la continuidad de la línea de investigación mediante el soporte económico. La presencia de los investigadores en jornadas organizadas por las propias familias y por las asociaciones permite esa cercanía que las familias y los pacientes necesitan, y suponen el recuerdo del fin último por el que trabajan los propios investigadores: mejorar el futuro y la calidad de vida de los pacientes. Los pacientes tienen mucho que decir en sus prioridades en investigación.

Como conclusión, podemos decir que estamos asistiendo a grandes e importantes cambios en cuanto a la situación de las enfermedades raras en todos los ámbitos. Prueba de ello es el crecimiento de su peso en las políticas nacionales e internacio- nales, el cambio de rol de los pacientes, médicos e investigadores y de sus relaciones recíprocas, la creciente sensibilización e implicación social, las mayores oportunidades por el momento tecnológico que vivimos Sin duda, muchos elementos que están evolucionando y creciendo pero que nos dejan a su vez muchas preguntas: ¿están los pacientes y las asociaciones asumiendo roles que deberían asumir las instituciones públicas?, ¿están los pacientes y las asociaciones sometiéndose a una exposición que no es conveniente? Y, por último y más importante, ¿serán estos cambios suficientes para determinar un verdadero punto de inflexión en la evolución de la investigación y los avances en enfermedades raras?

\section{AGRADECIMIENTOS}

La valiosa colaboración de Begonya Nafría, del Hospital Sant Joan de Déu, y de Jordi Cruz, de MPSFabry España, ha sido decisiva para la realización de este artículo.

\section{BIBLIOGRAFÍA}

Anderson, M., Elliott, E. J. y Zurynski, Y. A. (2013). Australian families living with rare disease: experiences of diagnosis, health services use and needs for psychosocial support. Orphanet Journal of Rare Diseases, 8, 22. https://doi. org/10.1186/1750-1172-8-22

Avellaneda Fernández, A., Layola, M., Izquierdo Martínez, M., Guilera, M., Badía Llach, X. y Ramón, J. R. (2007). Impacto sociosanitario en pacientes con enfermedades raras (estudio ERES). Medicina Clínica, 129 (17), pp. 646-651. https://doi.org/10.1157/13112096

Bañón Hernández, A. M., Fornieles Alcaraz, J., Solves Almela, J. A., Rius Sanchis, I. (coords.) (2011). Desafios y estrategias comunicativas de las enfermedades raras: la investigación médica como referente. Estudio cuantitativo-discursivo de la prensa escrita impresa y digital española (2009-2010). Valencia: Centro de Investigación Biomédica en Red de Enfermedades Raras CIBERER. Disponible en http://www.orphanet-espana. es/national/data/ES-ES/www/uploads/ investigacionmedica.pdf

Editorial: Making rare diseases a publichealth and research priority [Editorial]. The Lancet, 371 (9629) (14 de junio de 2008), p. 1972. https://doi. org/10.1016/S0140-6736(08)60842-9

Henderson, S. L., Packman, W. y Packman, S. (2009). Psychosocial aspects of patients with Niemann-Pick disease, type B. American Journal of Medical Genetics A 149 (11), pp. 2430-2436. https://doi. org/10.1002/ajmg.a.33077

Huete García, A., Díaz Velázquez, E., Díaz García, E. Sola Bautista, A., Petisco Rodríguez, E. y Lara Gonzalo, P. (2009). Estudio sobre situación de Necesidades Sociosanitarias de las personas con En- fermedades Raras en España. Estudio ENSERio. Madrid: Federación Española de Enfermedades Raras FEDER. Disponible en https://enfermedades-raras. org/images/stories/documentos/Estudio_ENSERio.pdf

Weely, S. van y Leufkens H. G. M. (2004). Orphan diseases. En: Priority Medicines for Europe and the World. A Public Health Approach to Innovation. Ginebra: World Health Organization, pp. 95-100. Disponible en http://www.who. int/medicines/areas/priority_medicines/BP6_19Rare.pdf

\section{Recursos de Internet}

Kaplan, W. y Laing, R. (2004). Priority Medicines for Europe and the World. Ginebra: World Health Organization. Disponible en http://apps.who.int/iris/bitstream/ handle/10665/68769/WHO_EDM_PAR 2004.7.pdf;jsessionid=D110B2A26E61 655CF14FA6D2C3A4C5BA? sequence $=1$ 